\footnotetext{
${ }^{10} \mathrm{H}$. Stanley, in Proceedings of the International Conference on Disordered Systems and Localization, edited by C. Di Castro (Springer-Verlag, Berlin, 1981), and references therein.
}

\author{
${ }^{11}$ Y. Gefen, A. Aharony, B. B. Mandelbrot, and \\ S. Kirkpatrick, Phys. Rev. Lett. 47, 1771 (1981). \\ ${ }^{12}$ R. B. Leibovitz, E. I. Alessandrini, and G. Deutsch- \\ er, Phys. Rev. B 25, 2965 (1982).
}

\title{
Critical Fields of the "Heavy-Fermion" Superconductor $\mathrm{CeCu}_{2} \mathrm{Si}_{2}$
}

\author{
U. Rauchschwalbe, W. Lieke, C. D. Bredl, and F. Steglich \\ Institut für Festkörperphysik, Technische Hochschule Darmstadt, D-6100 Darmstadt, West Germany
}

and

\author{
J. Aarts, ${ }^{(a)}$ K. M. Martini, and A. C. Mota ${ }^{(b)}$ \\ II. Physikalisches Institut, Universität zu Köln, D-5000 Köln 41, West Germany \\ (Received 12 April 1982)
}

\begin{abstract}
Measurements are reported of the lower and upper critical fields, $B_{c 1}(T)$ and $B_{c 2}(T)$, of $\mathrm{CeCu}_{2} \mathrm{Si}_{2}$. The observed, extremely high values of the slope $\left(-d B_{c_{2}} / d T\right)_{T_{c 0}}$ lend strong support to the formation of Cooper pairs by the heavy fermions which exist in the normal state of $\mathrm{CeCu}_{2} \mathrm{Si}_{2}$. Characteristic parameters of the system of heavy fermions are derived.
\end{abstract}

PACS numbers: 74.60.-w, 72.15.Qm, 74.70.Rv

Unusual superconducting materials, e.g., Chevrel phases, ${ }^{1}$ oxides, ${ }^{2}$ or organic conductors, ${ }^{3}$ have recently become of great interest in view of potential technical applications and the possibility of nonconventional mechanisms in superconductivity.

The (nearly) trivalent ternary compound $\mathrm{CeCu}_{2}-$ $\mathrm{Si}_{2}$ shows well-defined, localized magnetic moments above $T \cong 10 \mathrm{~K}$ (Ref. 4), but approaches a nonmagnetic state below $T \cong 10 \mathrm{~K}$ displaying the properties of a heavy Fermi liquid ${ }^{5}$; e.g., the specific heat was found to be $C \cong \gamma T$, where $\gamma$ $\cong 1 \mathrm{~J} \mathrm{~mole} \mathrm{~K}^{-1} \mathrm{~K}^{-2}$ is about a thousand times larger than for simple metals. $\mathrm{CeCu}_{2} \mathrm{Si}_{2}$ becomes superconducting below $T_{c} \cong 0.6 \mathrm{~K}$ (Ref. 5 ). The height of the specific-heat jump at $T_{c}$, comparable to the giant normal-state specific heat, $\gamma T_{c}$, has led to the conclusion ${ }^{5}$ that the superconducting state of $\mathrm{CeCu}_{2} \mathrm{Si}_{2}$ must be of a hitherto unknown kind, in that its Cooper pairs are formed by quasiparticles of very large effective mass (heavy fermions). In fact, the reference system $\mathrm{LaCu}_{2}-$ $\mathrm{Si}_{2}$, showing usual metallic behavior, does not become superconducting. ${ }^{5}$

To further support $\mathrm{CeCu}_{2} \mathrm{Si}_{2}$ being the first heavy-fermion superconductor, we present in this Letter results of the lower and upper critical fields, $B_{c 1}(T)$ and $B_{c 2}(T)$. Special emphasis has been put on the slope of $B_{c 2}(T)$ at $T_{c}$, which should reflect ${ }^{1}$ the high $\gamma$ coefficient. Analysis of these data will be used to estimate the key parameters of the normal Fermi-liquid state.

A wide scatter of $T_{c}$ 's has been reported for polycrystalline samples of $\mathrm{CeCu}_{2} \mathrm{Si}_{2}$, ranging from $<0.06 \mathrm{~K}$ (Ref. 6) to $0.65 \mathrm{~K}$ (Ref. 7). As was recently shown, ${ }^{8}$ however, $T_{c} \cong 0.55 \pm 0.15 \mathrm{~K}$ can always be achieved by powdering and subsequent proper heat treatment. On the other hand, no superconductivity has so far been observed for $\mathrm{CeCu}_{2} \mathrm{Si}_{2}$ single crystals. ${ }^{8,9}$ This might be due to a considerable ( $\cong 20 \%)$ deficiency in $\mathrm{Cu}$ occupation, as established for one of those single crystals. ${ }^{8}$ For the present investigations, two polycrystalline bulk samples were used. One of them (No. 7) was annealed at $1100{ }^{\circ} \mathrm{C}$ and found to be very clean, ${ }^{7}$ while the other one (No. 4), annealed at only $900{ }^{\circ} \mathrm{C}$, was less clean. ${ }^{10}$

Figure 1 shows the field dependence at constant temperatures of the magnetization $M$ for sample No. 7, which exhibits a static Meissner effect corresponding to $60 \%$ of the volume, when measured in powder form. ${ }^{7} M$ was measured by using a superconducting flux transformer between the sample and a flux-gate magnetometer (HewlettPackard Model 428B). In the inset, the initial slopes of these $M(B)$ curves are plotted as a function of temperature. The magnetization curves show broad maxima, probably caused by a broad distribution of demagnetizing fields within the polycrystalline sample. In such a situation, a lower 


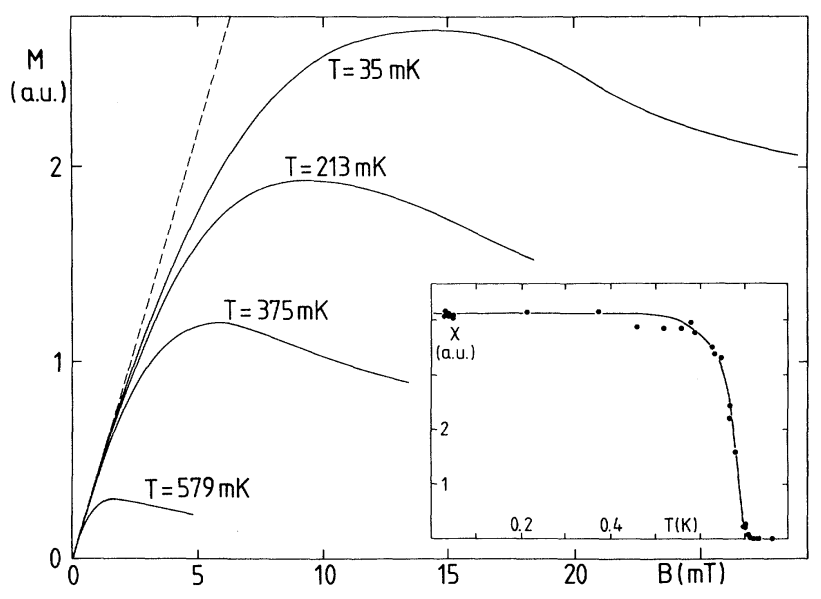

FIG. 1. de magnetization, $M$, of $\mathrm{CeCu}_{2} \mathrm{Si}_{2}$ (No. 7) as a function of the external magnetic field, $B$, at different temperatures. Inset shows low-field slopes of $M(B)$ curves vs temperature.

bound of the lower critical field is provided by $\boldsymbol{B}_{c 1} \cong \bar{B}_{c 1} /(1-D)$ where $\bar{B}_{c 1}$, plotted in the inset of Fig. 2, is the field at which the first deviation from the low-field linear $M(B)$ dependence occurs. The demagnetization factor, $D \cong 0.20$, of sample No. 7 was experimentally determined with a $\mathrm{Cd}$ sample of the same geometry $(4.1 \times 2.1 \times 2.0$ $\left.\mathrm{mm}^{3}\right)$. Figure 2 shows the temperature dependence of $B_{c_{2}}$ as determined from the midpoints of either inductive or calorimetric transitions for both samples.

We shall discuss the results on the clean $\mathrm{CeCu}_{2}$ $\mathrm{Si}_{2}$ sample No. 7 first. When the data in Fig. 2 are extrapolated to $T=0$, we find $\bar{B}_{c 1}(0) \simeq 1.8 \mathrm{mT}$, resulting in $B_{c 1}(0) \simeq 2.3 \mathrm{mT}$ and $B_{c 2}(0) \simeq 1.7 \mathrm{~T}$. This clearly indicates type-II behavior with a large Ginzburg-Landau (GL) parameter $\kappa$. For example, in proportion to the relatively low $T_{c}$, $B_{c 2}(0)$ is comparable to that of Chevrel-phase superconductors. ${ }^{1}$ An upward curvature is observed at low fields for both $B_{c 2}(T)$ and $B_{c 1}(T)$, presumably caused by inhomogeneities in the samples. For $B_{c 2}(T)$, we obtain from the linear region $\left(-d B_{c 2} / d T\right)_{T_{c 0}} \equiv B_{c 2}{ }^{\prime}=5.8 \mathrm{~T} / \mathrm{K}$, which is comparable to the highest values found for Chevrel-phase superconductors. ${ }^{1}$

In the following, we shall attempt to analyze this initial slope by using an expression which has been successfully applied to $A 15$ superconductors. ${ }^{11}$ Ignoring possible anisotropy effects in the polycrystalline $\mathrm{CeCu}_{2} \mathrm{Si}_{2}$ samples, we shall assume a spherical Fermi surface determined

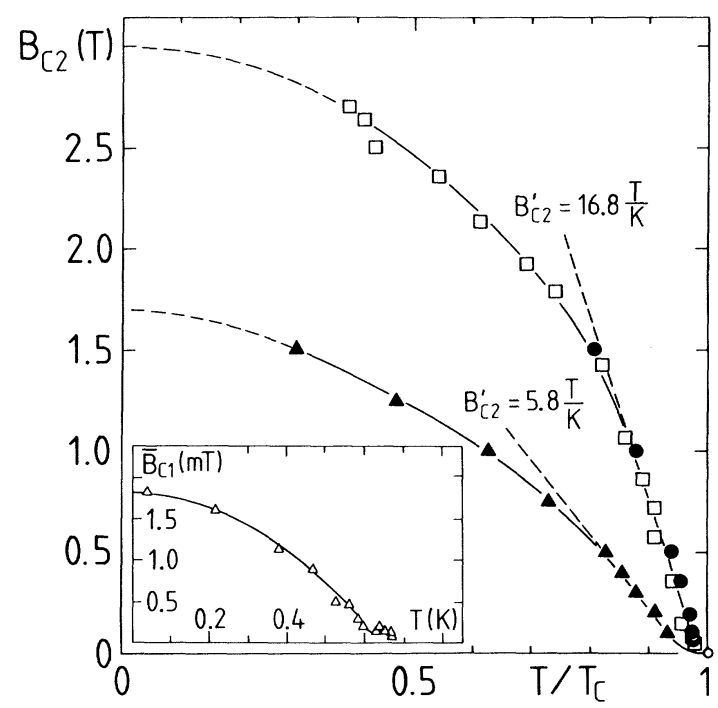

FIG. 2. Upper critical field, $B_{c 2}$, of $\mathrm{CeCu}_{2} \mathrm{Si}_{2}$ as a function of the reduced temperature, $T / T_{c}$. While $T_{c}$ is the transition temperature at $B_{c}=0$ as measured, $T_{c 0}$ is defined by extrapolation of linear $B_{c 2}(T)$ dependence to $\boldsymbol{B}=0$. Data were obtained from ac susceptibility (triangles: No. $7, T_{c 0}=0.64 \mathrm{~K}$; squares: No. 4 , $T_{c 0}=0.66 \mathrm{~K}$ ) or specific heat (circles: No. $4, T_{c 0}=0.56$ K). Inset shows $\vec{B}_{c 1}$ vs $T$ (No. 7) as explained in the text.

by a mean Fermi wave number $\bar{k}_{\mathrm{F}}$, yielding ${ }^{11}$

$$
\begin{aligned}
B_{c 2}{ }^{\prime} \simeq & \left(7.95 \times 10^{32} \frac{\mathrm{T} \mathrm{m}^{2} \mathrm{~K}^{2}}{\mathrm{~J}^{2}}\right) \frac{\gamma T_{\mathrm{c}}}{\bar{k}_{\mathrm{F}}{ }^{4}} \\
& +\left(4780 \frac{\mathrm{T} \mathrm{K} \mathrm{m}^{2}}{\Omega \mathrm{J}}\right) \gamma \rho_{0} .
\end{aligned}
$$

Inserting into Eq. (1) measured data for $T_{c}$ $(=0.64 \mathrm{~K})$, the residual resistivity $\rho_{0}\left(=3.5 \times 10^{-8}\right.$ $\Omega \mathrm{m}$ ), and the giant (heavy-fermion derived) coefficient $\gamma\left(\simeq 2.0 \times 10^{4} \mathrm{~J} \mathrm{~K}^{-2} \mathrm{~m}^{-3}\right.$, with $V_{\text {mole }} \simeq 5.03$ $\times 10^{-5} \mathrm{~m}^{3}$ ), we obtain $\bar{k}_{\mathrm{F}} \simeq 1.7 \times 10^{10} \mathrm{~m}^{-1}$. Probably because of anisotropy effects, this is slightly larger than $\bar{k}_{\mathrm{F}} \simeq 1.6 \times 10^{10} \mathrm{~m}^{-1}$ of the ordinary conduction-electron gas as previously estimated from the maximum high-temperature resistivity. ${ }^{12}$ The latter $\bar{k}_{\mathrm{F}}$ value corresponds to a reasonable valence-electron concentration of about 2 /atom. We conclude that both the ordinary conductionelectron gas at high temperature and the lowtemperature Fermi-liquid phase can be described by similar mean values of the Fermi wave number. This strongly suggests a description of the Fermi-liquid phase in $\mathrm{CeCu}_{2} \mathrm{Si}_{2}$ in the spirit of Landau's phenomenological theory, ${ }^{13}$ i.e., by assuming some strong interaction between con- 
duction electrons which leaves the Fermi wave number unchanged but dramatically renormalizes the properties of the conduction-electron states near $k_{\mathrm{F}}$. For example, the Fermi velocity of the quasiparticles, $\bar{v}_{\mathrm{F}} \simeq\left(6.02 \times 10^{-13} \mathrm{~J} \mathrm{~K}^{-2} \mathrm{~s}^{-1}\right) \bar{k}_{\mathrm{F}}^{2} \gamma^{-1}$ $\simeq 8.7 \times 10^{3} \mathrm{~m} \mathrm{~s}^{-1}$, and their effective mass, $m^{*}$ $=\hbar \bar{k}_{\mathrm{F}} \bar{v}_{\mathrm{F}}^{-1} \simeq 220 m_{0}$, differ by two orders of magnitude from the corresponding free-electron values. We wish to stress that, because of the measured $\boldsymbol{B}_{c 2}{ }^{\prime}$ value, this Fermi-liquid phase cannot be attributed to a narrow $4 f$ band originating from one tightly bound electron per $\mathrm{Ce}$ ion, ${ }^{14}$ since this would imply a much too small $\bar{k}_{\mathrm{F}}$, i.e., $\simeq 0.7$ $\times 10^{10} \mathrm{~m}^{-1}$ (associated with $\bar{v}_{\mathrm{F}} \simeq 1.5 \times 10^{3} \mathrm{~m} \mathrm{~s}^{-1}$ and $\left.m^{*} \simeq 530 m_{0}\right)$.

The estimation of some important parameters, which characterize the novel superconducting state of $\mathrm{CeCu}_{2} \mathrm{Si}_{2}$, is also straightforward. Using relations given in Ref. 11 and the above values for $T_{c}, \rho_{0}$ and $\gamma$, we find the BCS coherence length $\xi_{0} \simeq 1.9 \times 10^{-8} \mathrm{~m}$. This is comparable to the mean free path of the quasiparticles, $l \simeq 1.2$ $\times 10^{-8} \mathrm{~m}$. The London penetration depth (as $T-0$ ) assumes an unusually high value, i.e., $\lambda \simeq 2 \times 10^{-7}$ m. The GL parameter is estimated to be $\kappa \simeq 22$ for sample No. 7 and $\simeq 10$ in the "pure limit" $(l$ $\left.\gg \xi_{0}\right)$.

With use of this $\kappa$ value, the above analysis of the initial slope of $B_{c 2}(T)$ can now be supported by the calculation of certain quantities for sample No. 7 and comparing them with the corresponding quantities as either directly measured or calculated from the results of other experiments. For this purpose, we first estimate ${ }^{1}$ the "orbital critical field" (as $T \rightarrow 0$ ), i.e., $B_{c 2} *(0) \simeq 0.69 B_{c 2}$ ' $\times T_{c} \simeq 2.6 \mathrm{~T}$. This is about $50 \%$ higher than $B_{c 2}(0)$ as measured, pointing to the presence of other pair-breaking mechanisms like Pauli paramagnetic limiting or exchange scattering from paramagnetic impurities. Now we can estimate the thermodynamic critical field (as $T \rightarrow 0$ ) from (i) $B_{c 2} *(0)$ and (ii) the specific-heat coefficient $\gamma$ [assuming a parabolic $B_{c t h}(T)$ dependence]. We find almost the same values, namely (i) $B_{c t h}(0)$ $=B_{c 2} *(0) / \sqrt{2} \kappa_{1}(0) \simeq 66 \mathrm{mT}\left[\right.$ with $\kappa_{1}(0) \simeq 1.26 \kappa$ (Ref. 15)] and (ii) $B_{c \text { th }}(0)=\left[7.65 \times 10^{-4}\left(\mathrm{~m}^{3} / \mathrm{J}\right)^{1 / 2}\right] \gamma^{1 / 2} T_{c}$ $\simeq 69 \mathrm{mT}$ (Ref. 16). This is much higher than $B_{c t h}(0) \simeq 3 \mathrm{mT}$ of the conventional superconductor Cd with comparable $T_{c}$. Since $B_{c t h}(0)$ determines the "condensation energy" of a superconductor, we find the superconducting state of $\mathrm{CeCu}_{2} \mathrm{Si}_{2}$ to be of much higher thermodynamic stability than its conventional counterpart. This is caused by the extremely high density of Cooper-pair states, which tracks the giant $\gamma$ coefficient, in the former material.

With $\boldsymbol{B}_{c t h}(0)$ and $\kappa$ we can also estimate the lower critical field through $B_{c \mathrm{r}}(0)=B_{c \mathrm{th}}(0) \ln \kappa_{3}(0) /$ $\sqrt{2} \kappa_{3}(0) \simeq 6 \mathrm{mT}$, where $\kappa_{3}(0)=1.15 \kappa$ was used. ${ }^{15}$ $B_{c 1}(0)$ agrees within an order of magnitude with the measured value $(\simeq 2.3 \mathrm{mT})$, which may be considered to be satisfying enough, especially if one keeps in mind the difficulties in measuring $B_{c 1}(0)$.

Finally, we are able to estimate the size of the specific-heat jump at $T_{c}$, namely $\Delta C \simeq\left(6.86 \times 10^{5}\right.$ $\left.\mathrm{J} \mathrm{T}^{-2} \mathrm{~m}^{-3}\right)\left(2 \kappa^{2}-1\right)^{-1} T_{c} B_{c 2}{ }^{2} \simeq 1.53 \times 10^{4} \mathrm{~J} \mathrm{~K}^{-1} \mathrm{~m}^{-3}$, which is very close to the experimental value, $\Delta C=1.59 \times 10^{4} \mathrm{~J} \mathrm{~K}^{-1} \mathrm{~m}^{-3}$ (Ref. 7). These thermodynamic relations give strong evidence that the Fermi-liquid phase of $\mathrm{CeCu}_{2} \mathrm{Si}_{2}$ is formed by renormalized conduction-electron states in the vicinity of $\bar{k}_{\mathrm{F}}=(1.6-1.7) \times 10^{10} \mathrm{~m}^{-1}$, and they dis prove, again, the picture of one $4 f$-derived heavy fermion per Ce ion ${ }^{14}$; for in this case $\bar{k}_{\mathrm{F}} \simeq 0.7$ $\times 10^{10} \mathrm{~m}^{-1}$ results in $\xi_{0} \simeq 3 \times 10^{-9} \mathrm{~m}, \lambda \simeq 3 \times 10^{-7}$ $\mathrm{m}$, and $\kappa \simeq 100$, which is much too large a value.

Having found consistency in the various results for the pure sample No. 7, we now turn to the $B_{c 2}$ data of sample No. 4. As is shown in Fig. 2, the initial slope of $B_{c 2}(T)$ is $16.8 \mathrm{~T} / \mathrm{K}$ for this sample, the highest value observed for any superconductor. From the residual resistivity, $\rho_{0}$ $\leqslant 4 \times 10^{-7} \Omega \mathrm{m}$ (Ref. 17), the mean free path of sample No. 4 is estimated to be much smaller than the coherence length, i.e., sample No. 4 clearly represents the "dirty limit." Using the expression for $B_{c 2}$ ' in the "dirty limit," ${ }^{11} B_{c 2}$ ' $=\left(4.48 \times 10^{3} \mathrm{~T} \mathrm{~K} \mathrm{~m}^{2} \mathrm{~J}^{-1} \Omega^{-1}\right) \gamma \rho_{0}$, with $\gamma=1.4 \times 10^{4}$ $\mathrm{J} \mathrm{K}^{-2} \mathrm{~m}^{-3}$ (Ref. 10), we estimate $B_{c 2}{ }^{\prime} \leqslant 25 \mathrm{~T} / \mathrm{K}$. Again, there is satisfactory agreement with the experimental result. ${ }^{18}$

To conclude, we have found that (i) the purer $\mathrm{CeCu}_{2} \mathrm{Si}_{2}$ sample shows an initial slope of the upper critical field $B_{c 2}(T)$ of the same size $(\simeq 6$ $\mathrm{T} / \mathrm{K})$ as $B_{c 2}{ }^{\prime}$ of the best high-field superconductors (with much higher transition temperatures) known so far; this is caused by the very small Fermi velocity of the heavy fermions forming the Cooper pairs in $\mathrm{CeCu}_{2} \mathrm{Si}_{2}$ [in the "pure limit" $B_{c 2}{ }^{\prime} \sim T_{c} / v_{\mathrm{F}}^{2}$, first term in Eq. (1)]; (ii) a decrease of the quasiparticle mean free path results in a further increase of $B_{c 2}{ }^{\prime}$ to the record value of $\simeq 17 \mathrm{~T} / \mathrm{K}$, which is due to an additional contribution [second term in Eq. (1), $\sim\left(l v_{\mathrm{F}}\right)^{-1}$ ]; (iii) surprisingly enough, possible anisotropy effects, ${ }^{19}$ which might originate from the quasi two-dimensional structure of $\mathrm{CeCu}_{2} \mathrm{Si}_{2}$, do not 
dominate $B_{c_{2}}(T)$ in the polycrystalline samples studied, for the reduced specific-heat-jump height is of the order of the BCS value in either case $\mathrm{e}^{7,10}$ and, in addition, the "dirtier," i.e., more isotropic, sample shows the higher $B_{c 2}$ ' value [providing an a posteriori justification of the assumption of a spherical Fermi surface made when using Eq. (1) ]; (iv) the low-temperature Fermiliquid phase of $\mathrm{CeCu}_{2} \mathrm{Si}_{2}$ is described by a Fermi wave number close to that of the ordinary conduction-electron gas.

The physical origin of both the formation of the extremely heavy fermions and the attractive interaction between the fermions, which constitutes the novel superconducting state of $\mathrm{CeCu}_{2} \mathrm{Si}_{2}$, remains unknown.

One of us (F.S.) should like to acknowledge stimulating discussions with D. Rainer, R. A. Klemm, $\varnothing$. Fischer, E. Müller-Hartmann and P. Entel, and one of us (A.C.M.) with K. Kwasnitza and D. Wohlleben. This work was supported by Sonderforschungsbereiche 65 and 125 of the Deutsche Forschungsgemeinschaft.

\footnotetext{
(a)Present address: Natuurkundig Laboratorium, Universiteit van Amsterdam, NL-1018 XE Amsterdam, The Netherlands.

(b) Present address: Laboratorium für Festkörperphysik, Eidgenössiche Technische Hochschule, CH-8093 Zürich, Switzerland.

${ }^{1}$ For a review, see $\varnothing$. Fischer, Appl. Phys. 16, 1 (1978).

${ }^{2}$ See, e.g., A. W. Sleight, J. L. Gillson, and P. E. Bierstedt, Solid State Commun. 17, 27 (1975).

${ }^{3}$ D. Jerome, A. Mazaud, M. Ribault, and K. Bechgaard, J. Phys. (Paris), Lett. 41, L95 (1980).

${ }^{4}$ S. Horn, M. Loewenhaupt, E. Holland-Moritz,
}

F. Steglich, H. Scheuer, A. Benoit, and J. Flouquet, Phys. Rev. B 23, 3171 (1981).

${ }^{5}$ F. Steglich, J. Aarts, C. D. Bredl, W. Lieke, D. Meschede, W. Franz, and H. Schäfer, Phys. Rev. Lett. $\underline{43}, 1892$ (1979).

${ }^{6}$ G. W. Hull, J. H. Wernick, T. H. Geballe, J. V. Waszczak, and J. E. Bernardini, Phys. Rev. B 24, 6715 (1981).

${ }^{7}$ W. Lieke, U. Rauchschwalbe, C. D. Bredl, F. Steglich, J. Aarts, and F. R. de Boer, J. Appl. Phys. 3ㅜ, 2111 (1982).

${ }^{8}$ C. D. Bredl, H. Spille, U. Rauchschwalbe, W. Lieke, F. Steglich, G. Cordier, W. Assmus, M. Herrmann, and J. Aarts, in Proceedings of the International Conference on Magnetism, Kyoto, 1982 (to be published).

${ }^{9}$ F. G. Aliev, N. B. Brandt, R. B. Wociev, V. V. Moshtaukov, and S. M. Chubinov, Pis'ma Zh. Eksp. Teor. Fiz. 35, 435 (1982).

${ }^{10}$ F. Steglich, J. Aarts, C. D. Bredl, W. Lieke, D. Meschede, W. Franz, and H. Schäfer, J. Magn. Magn. Mater. 15-18, 889 (1980).

${ }^{11}$ T. P. Orlando, E. J. McNiff, Jr., S. Foner, and M. R. Beasley, Phys. Rev。B 19, 4545 (1979).

${ }^{12}$ W. Franz, A. Griessel, F. Steglich, and D. Wohlleben, Z. Phys. B 31, 7 (1978).

${ }^{13} \mathrm{P}$. Nozières, Theory of Interacting Fermi Systems (Benjamin, New York, 1964).

${ }^{14}$ K. Andres, J. E. Graebner, and H. R. Ott, Phys. Rev. Lett. 35, 1779 (1975).

${ }^{15} \mathrm{~B}$. Serin, in Superconductivity, edited by R. D. Parks (Marcel Dekker, New York, 1969), Vol. 2, p. 9.

${ }^{16}$ R. R. Hake, Phys. Rev. 158, 356 (1966).

${ }^{17}$ Whereas $B_{c 2}(T)$ of sample No. 4 was determined after annealing at $900{ }^{\circ} \mathrm{C}$, the resistivity was measured in the unannealed state only and, therefore, can be taken only as an upper bound of $\rho_{0}$ for the annealed sample.

${ }^{18}$ From $B_{c 2}{ }^{\prime}=16.8 \mathrm{~T} / \mathrm{K}$ we would expect the residual resistivity of the $\left(900^{\circ} \mathrm{C}\right)$ annealed sample No. 4 to be $\rho_{0} \simeq 2.7 \times 10^{-7} \Omega \mathrm{m}$, quite a reasonable value.

${ }^{19}$ See, e.g., M. Ikebe, K. Katagiri, N. Noto, and Y. Muto, Physica (Utrecht) 99B, 209 (1980); P. Entel and M. Peter, J. Low Temp. Phys. 22, 613 (1976). 